\title{
Quantitative Analysis of Alcoholic Drinks. Use of Calibration Curve Method to Determine the Alcoholic Degree of Samples of Paesanella, a Distillate of the Family of Grappa
}

\author{
Checchetti Andrea* \\ Istituto d’Istruzione Superiore “Leonardo da Vinci”, San Giovanni in Fiore, Italy \\ *Corresponding author: andreachecchetti@alice.it \\ Received April 15, 2015; Revised May 18, 2015; Accepted May 27, 2015
}

\begin{abstract}
Within the framework of laboratory activities relating to the teaching programof a fifth-year class of a technical school in Italy, we have determined, by the means of a spectrophotometer UV/vis, the alcoholic degree of a series of samples of Paesanella, a distillate of the family of grappa. In this experiment of quantitative analysis the students prepared anaqueous solution of ethanol to draw, by means of measurements of absorbance, the calibration line. The students found, making use of EXCEL, the linear equation with which to calculate the alcoholic degree of the samples of Paesanella.
\end{abstract}

Keywords: Spirit, "Grappa”, "Paesanella”, alcoholic degree, calibration line, UV/vis spectroscopy

Cite This Article: Checchetti Andrea, "Quantitative Analysis of Alcoholic Drinks. Use of Calibration Curve Method to Determine the Alcoholic Degree of Samples of Paesanella, a Distillate of the Family of Grappa." World Journal of Chemical Education, vol. 3, no. 3 (2015): 70-73. doi: 10.12691/wjce-3-3-3.

\section{Introduction}

The author, currently attending a masters program on Teaching Science, sponsored by the Italian Ministry of Education. The activity is part of a laboratorial learning path, the main objective of which is to facilitate and improve science learning in schools. With the help of the laboratorial methodology, the tutorials constitute a valid and mutual integration with the theoretical part; not only as a moment of experimental verification of what was learned in theory, but also as a tool to derive laws, principles and theoretical models starting from experiences. The lessons have the aim of giving the students a different approach to Chemistry in order to promote reflection, combining the acquired knowledge and skills with the problems of everyday life. For this reason, laboratory work concerns topics such as water chemistry, the environment, food, materials and daily life.

The general objectives are:

1. Develop activities and related documentation suitable to the didactic needs of the secondary schools of second degree;

2. Contribute with the use of the scientific laboratory to the recovery and deepening of the school curriculum, moving from knowledge to know-how.

The training of the students involves the construction of a series of operational steps

- Theoretical and practical insights, propaedeutic to the performance of laboratory activities;
- $\quad$ Use of scientific tools available in the school;

- Implementation of a series of experiments, documenting the activities in order to insert them in learning units of Chemistry;

- Acquisition of the essential skills needed in the laboratory, intended not only as a simple sequence of manual operations, but as a practical implementation of planning skills backed by adequate processing autonomy.

It is important to remember that the laboratory activities are evaluated with respect to:

- Basic knowledge of analytical principles and their stoichiometric calculations;

- Organizational skills of students in designing and carrying out the analysis;

- Ability to record the experimental data obtained from the experiment;

- Validity of the results obtained.

\section{Experimental Procedure}

\subsection{Method}

The name "Grappa" is used exclusively to indicate the spirits obtained from raw materials, derived from grapes produced and vinified in Italy, distilled and bottled in plants located throughout the country. The distilled grappa is obtained directly in the still by means of water vapor or through the addition of water to fermented or semifermented marcs.

At the end of the distillation you get a brandy of high alcoholic strength (typically between 65 and 86\% 
(alc./Vol.) and, therefore, not yet suitable to be consumed and appreciated. The next step is then to reduce the percentage of alcohol that, by law [1], cannot be less than $37.5 \%$ Vol.

The production of grappa, as documented in several historical evidences, is closely related to the area of origin. According to tradition, within the territory of San Giovanni in Fiore, situated in the Sila (Calabria, Italy), the typical alcoholic drink produced locally is the Paesanella, a distillate of the family of grappa. Its peculiarity is that it is homemade, outside of any industrial logic.

The fundamental law that governs the quantitative spectrophotometric analysis is the Lambert-Beer law. Mathematically, Lambert-Beer law is expressed as

$$
\mathrm{A}=a b \mathrm{C}
$$

Where

$$
\begin{aligned}
& \text { A = absorbance } \\
& a=\text { absorptivity coefficient } \\
& \mathrm{b}=\text { path length of radiation through sample }(\mathrm{cm}) \\
& \mathrm{C}=\text { concentration of water in mixture }
\end{aligned}
$$

Both $a$ and $b$ are constant, so $a$ is directly proportional to the concentration $\mathrm{C}$.

Among the most used methods to determine the unknown concentration of an analyte there is the calibration line [2].

This method, in general, is suited to spectrophotometric techniques. The method involves a series of operations that can be synthetically summarized:

1. Preparation of a standard concentrated solution of the analyte;

2. Preparation of a series of standard solutions by diluting the concentrated standard solution in volumetric flasks;

3. Preparation of the solution that contains all the reagents with the exception of the analyte (reagent blank);

4. Set the spectrophotometer for measurement, by suitably choosing the values for the operating variables;

5. Recording of instrumental responses of each standard solution and blank reagent;

6. Construction of a calibration graph by placing on the abscissa the concentration of the standards and the absorbance on the ordinate;

7. If the graph is a straight line you can determine the equation by making use of Excel.

\subsection{Principles}

Near-infrared light generally refers to light with wavelengths from 800 to $2.5 \mathrm{~nm}$ as shown in Figure 1. Near-infrared light absorption is weak since the light absorption is based overtones and combined tones in midinfrared light region. Aqueous solutions are a type of solution that can be measured in the near infrared region [3].

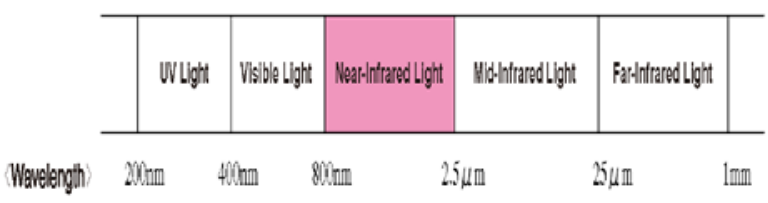

Figure 1. Near-Infrared Light Region
The water molecule shows an overtone absorption in the near-infrared in the range between 970 and $980 \mathrm{~nm}$ $[3,4]$. When it is mixed with substances such as ethanol, which do not exhibit absorption in this range, the absorbance measured is proportional to its concentration in the mixture.

Therefore, it is possible to obtain a calibration line which allows the determination of the alcoholic degree of drinks based on the determination of the amount of water they contain.

\subsection{Apparatus and reagents}

The absorbance measurements were made on Jasco 660 UV/vis spectrophotometer.

Quantification of water using spectrophotometer may be carried out by preparing mixture and measuring its absorbance at suitable wavelength. The wavelength normally selected is that of maximum absorption $\left(\lambda_{\max }\right)$.

Ethanol was obtained from Carlo Erba reagents (Italy).

\subsection{Procedure}

Opportune amounts of ethanol (mL) were mixed to water in a series of $50 \mathrm{ml}$ of volumetric flasks. Distilled water used was from an all glass still. Table 1 shows the percentages of ethanol and the relative absorbance measured to $974 \mathrm{~nm}$, using cuvette of $1 \mathrm{~cm}$. Standard solutions were scanned in UV spectrophotometer between 800 to $1100 \mathrm{~nm}$ on spectrum mode using ethanol as blank. Water shows $\lambda_{\max }$ to $974 \mathrm{~nm}$ (Figure 2). All the analytical data were stored in a personal computer and analyzed using Spectra Manager II software (Jasco, UK).

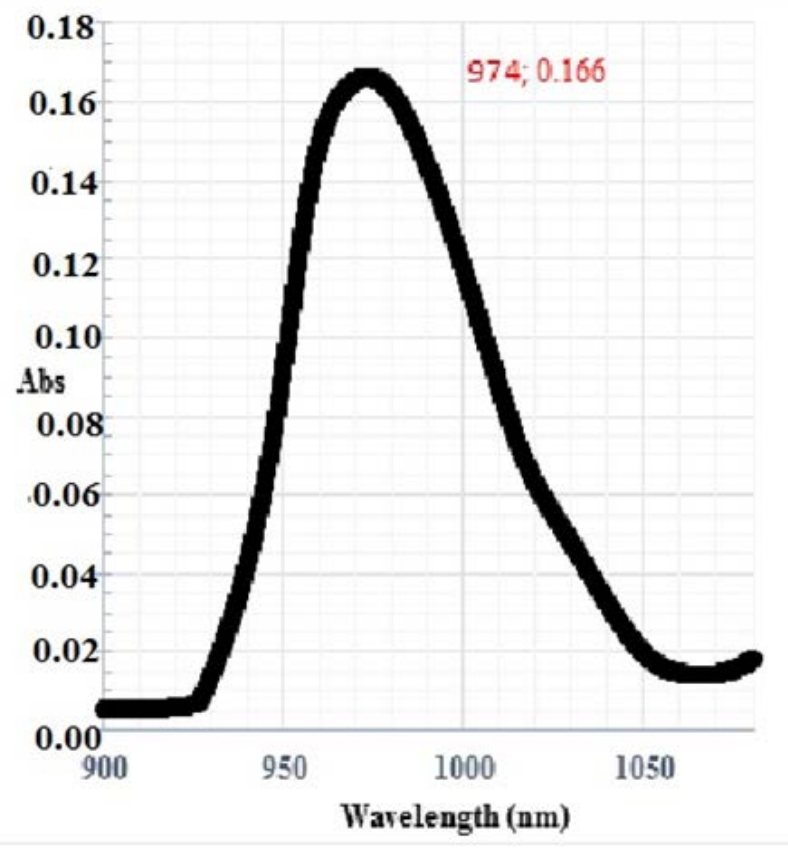

Figure 2. Absorption spectrum of water

\section{Results and Discussion}

\subsection{Curve of Calibration}

A seven point calibration curve was obtained in a concentration range from 0 to $100 \%$ for ethanol. The 
response of ethanol was found to be linear in the investigation concentration range and the linear regression equation was

$$
y=-0,0017 x+0.161
$$

with correlation coefficient 0.9947 (Table 1, Figure 3).

Table 1. Absorbance values vs percentage of ethanol

\begin{tabular}{|l|l|}
\hline Ethanol (\%) & Absorbance \\
\hline 0 & 0.166 \\
\hline 10 & 0.139 \\
\hline 20 & 0.130 \\
\hline 40 & 0.098 \\
\hline 60 & 0.058 \\
\hline 80 & 0.022 \\
\hline 90 & 0.014 \\
\hline 100 & 0 \\
\hline
\end{tabular}

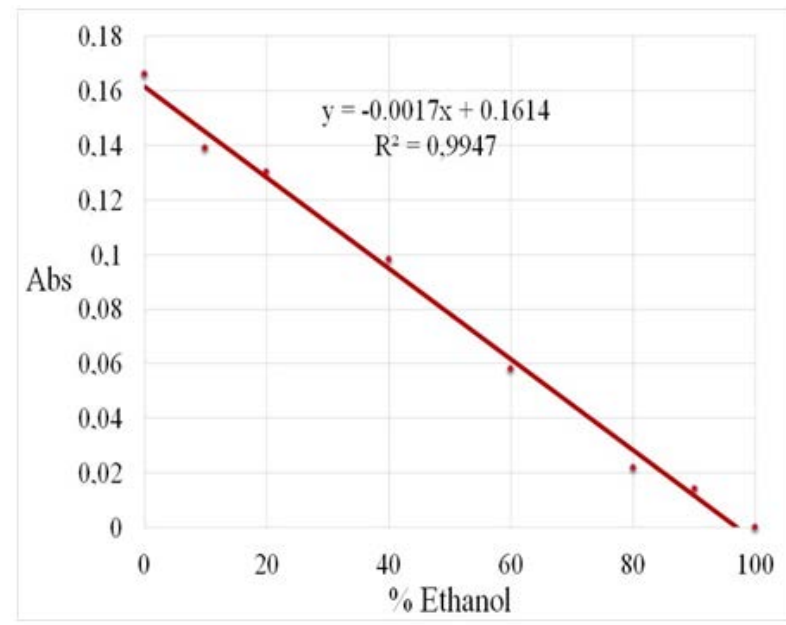

Figure 3. Calibration line

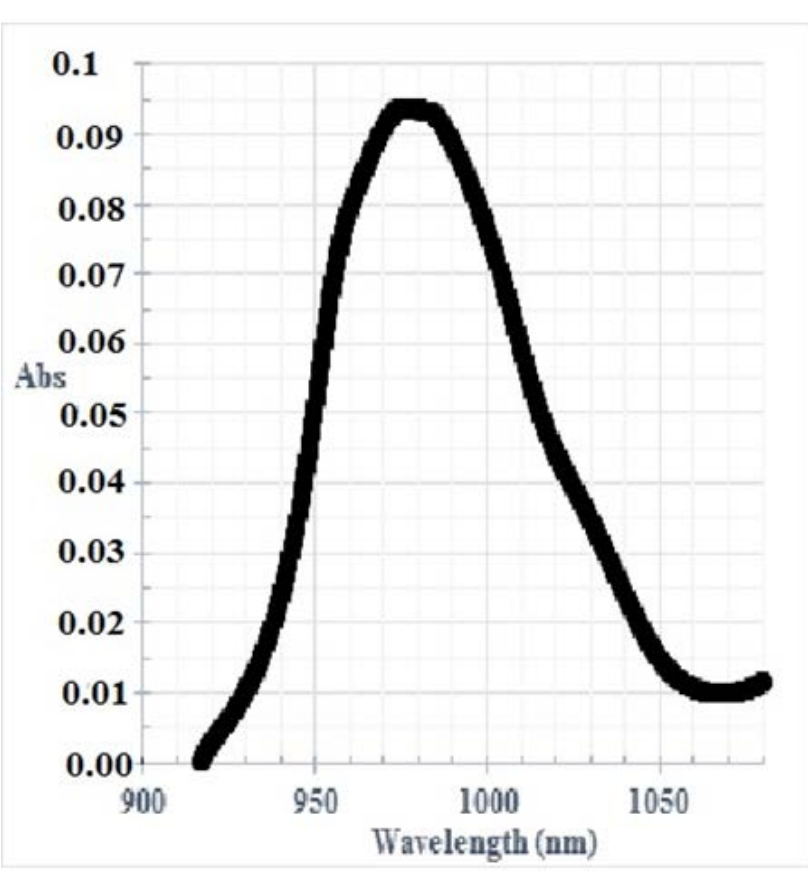

Figure 4. Absorption spectrum of cognac

Before measuring the absorbance of samples of Paesanella, a sample of Gran Marnier cognac was used, the label of which reported a volume concentration of $40 \%$. Figure 4 shows the spectrum and the measured absorbance to $\lambda_{\max }$ was 0.0937 with a volume concentration of $39.82 \%$. The relative error was of $0.44 \%$.

\subsection{Alcoholic Degree of Paesanella}

Table 2 shows the results obtained for four samples of Paesanella. Figure 5 shows the related spectra.

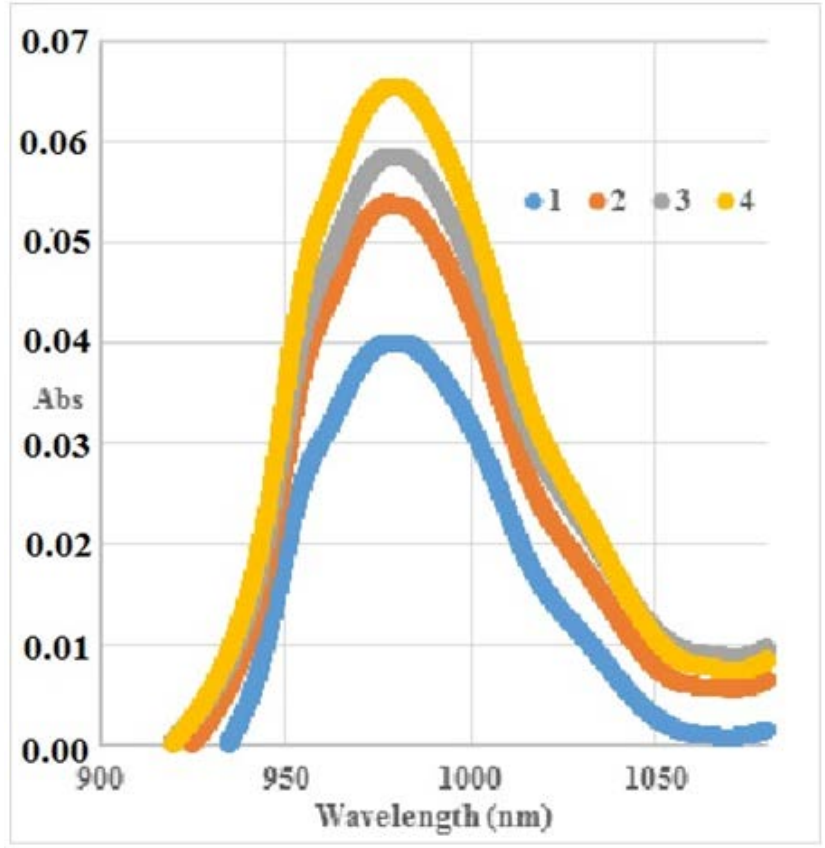

Figure 5. Absorption spectra of samples of Paesanella

Table 2. Alcoholic degree of samples of Paesanella

\begin{tabular}{|c|c|c|}
\hline Paesanella & Alcoholic degree & Absorbance \\
\hline n. 1 & 71.41 & 0.040 \\
\hline n. 2 & 63.76 & 0.053 \\
\hline n. 3 & 60.82 & 0.058 \\
\hline n. 4 & 56.71 & 0.065 \\
\hline
\end{tabular}

\section{Conclusion}

Over the last years it has become a fundamental laboratory practice to allow students to be active protagonist of their know-how.

The results of the latest reports OECD-PISA [5] of Italian students demonstrate how the models of transmission of scientific knowledge are no longer sufficient and that it is urgent to introduce new teaching methodologies. The implementation of lab activities allows us to acquire an set of key skills.

In this direction we have tried to bring together two visions of conceiving the laboratory:

- The first as a space-time verification of the laws;

- The second as fertile ground for developing critical thinking.

In this way the student is able:

- to merge the manual skills with mental ones;

- to create the right synergies between thinking and acting, in order to share theories and concepts with the elaboration and the experimental proceed. 
The laboratory didactics is therefore an instrument of strong innovation to be introduced in the educational chain that goes from primary to secondary school.

Here we have proposed a type of laboratorial activity developed within the teaching of "Instrumental Chemical Analysis". In particular we have determined the alcoholic degree of four distillates derived from marc, called Paesanella. The alcoholic degree was obtained by making use of the calibration curve absorbance/percentage of ethanol $(\mathrm{V} / \mathrm{V})$. The ethanol percentages change between 72 and $55 \%(\mathrm{~V} / \mathrm{V})$.

\section{Acknowledgement}

The author thanks Prof. Annamaria Secreti and Prof. Donato Martano from I.I.S. "Leonardo da Vinci” of San
Giovanni in Fiore (Italy) for their help. This work was supported by theInstitute of Higher Education "Leonardo da Vinci” of San Giovanni in Fiore (Italy).

\section{References}

[1] Ministero delle Politiche Agrigole Alimentari e forestali, Scheda Tecnica della grappa, Decreto 1 August 2011.

[2] R. Cozzi, P. Protti, T. Ruaro, Analisi Chimica Strumentale. Bologna, Italy, Zanichelli, 1997, vol. C.

[3] H. Abo, Near-Infrared Region Measurement and Related Considerations Part 1 FTIR Talk Letter Vol. 9.

[4] R. Cozzi, P. Protti, T. Ruaro, Analisi Chimica Strumentale. Bologna, Italy, Zanichelli, 1997, vol. B.

[5] PISA 2009 Results Executive Summary Available: http://www.oecd.org/dataoecd/34/60/46619703.pdf. 